\title{
Concurrent cytologic and colposcopic evaluation of symptomatic cervical erosion
}

\author{
Shreya Goel*, Prabha Lal
}

Department of Obstetrics and Gynaecology, Lady Hardinge Medical College, Delhi, India

Received: 11 November 2021

Revised: 02 December 2021

Accepted: 03 December 2021

\section{*Correspondence:}

Dr. Shreya Goel,

E-mail: shreya.goel1234@gmail.com

Copyright: () the author(s), publisher and licensee Medip Academy. This is an open-access article distributed under the terms of the Creative Commons Attribution Non-Commercial License, which permits unrestricted non-commercial use, distribution, and reproduction in any medium, provided the original work is properly cited.

\begin{abstract}
Background: Cervical cancer is amongst the leading causes of deaths due to cancer in developing countries. Moreover, preinvasive lesions of the cervix have a long latency period for conversion into malignancy and are also detectable by screening techniques. Hence, colposcopy in addition to cytology should be carried out wherever facility is available to ensure early detection and timely management.

Methods: Simultaneous cytology and colposcopy was done for 80 women with symptomatic cervical erosion followed by a colposcopic directed biopsy in women with MRCI >3. Finally, correlation between cytology, colposcopy and histopathological results was done.

Results: 65/80 women were biopsied. 12/80 women had MRCI >6 amongst which 10/80 were confirmed to have a high grade lesion on histopathology. 13/80 had lesser abnormalities (ASCUS and LSIL) amongst which 3/80 had CIN1 on histopathology. Only $2 / 80$ had HSIL on cytology as compared to 8/80 on histopathology that had CIN 2/3. Lastly, only $1 / 80$ had SCC on cytology compared to $2 / 80$ on histopathology. The sensitivity, specificity, PPV and NPV of cytology and colposcopy for diagnosing cervical dysplasia was $46.1 \%, 83.5 \%, 35.2 \%, 88.8 \%$ and $84.6 \%$, $86.5 \%, 55 \%, 96.6 \%$ respectively making colposcopy a better screening tool than cytology for evaluating cervical malignancy.

Conclusions: Colposcopic examination should ideally be carried out in all women with symptomatic cervical erosion in addition to cytology. Moreover, suspicious areas should be biopsied even if cytology is normal to exclude malignancy.
\end{abstract}

Keywords: Erosion, Cytology, Colposcopy, Malignancy

\section{INTRODUCTION}

Cervical cancer is the second most common cancer affecting women worldwide and is particularly responsible for deaths due to cancer in developing countries. 67,477 deaths were reported amongst 1,22,844 diagnosed with cervical cancer in India. ${ }^{1}$ The most common etiological factors responsible for cervical cancer include sexually transmitted viral infections (human papilloma virus, human immunodeficiency virus and herpes simplex virus). Women with multiple sexual partners, early age at first intercourse and increasing parity are particularly vulnerable. ${ }^{2}$ The present guidelines for the screening of cervical cancer recommend an early Pap Smear for all sexually active women in their first hospital visit itself. Any abnormality on pap smear is followed up with the help of colposcopy. A colposcopic directed biopsy is then used for the histopathological analysis of the abnormal areas seen on colposcopy which is the gold standard for diagnosis of cervical malignancy. This leads to a delay in the diagnosis as well as early treatment of the premalignant lesions. In colposcopy, the 
most common grading used is Reid's colposcopic index. This includes five colposcopic signs (thickness, colour, surface contour, vascular atypia, and iodine staining), each given a score of zero to two. ${ }^{3}$ Nowadays, thickness and contour is replaced by sharpness of margins in the new modified Reid's index which is found to be more useful. A newer scoring system, the Swede Score has also been developed. Since preinvasive lesions can be detected timely by effective screening procedures in place and the disease has a high latency period for conversion into malignancy, early detection and timely management should be done. The concurrent use of cytology and colposcopy has not yet been studied extensively and given the high sensitivity of colposcopy along with a high specificity of cytology, a combination would allow premalignant lesions of the cervix to be detected early and managed timely.

\section{METHODS}

\section{Study type, location and duration}

Current study is a prospective cross-sectional study conducted at department of obstetrics and gynaecology in association with the department of pathology in Lady Hardinge medical college and associated hospitals from November 2018 to March 2020.

\section{Inclusion criteria}

All women aged 21-50 years having symptoms of either vaginal discharge, post coital bleeding, intermenstrual bleeding or abnormal uterine bleeding with cervical erosion on per-speculum examination formed our inclusion criteria. 80 such women with symptomatic cervical erosion were enrolled in the present study. Pregnant women, women on oral contraceptive pills and known cases of premalignant/malignant lesions of cervix were excluded from the study.

\section{Methodology}

A detailed history including age at first intercourse, personal hygiene, socioeconomic status, parity and sexual practices was taken and a careful examination was done for all the cases. A pap smear was taken for all cases and reporting was done according to Bethesda's classification followed by colposcopic examination. All women were classified according to the modified Reid's colposcopic index into 3 categories and colposcopic directed biopsy was done for women with MRCI >3. Colposcopic signs such as Ridge sign, Rag sign and inner border sign were also studied. Finally, correlation between cytology, colposcopy and histopathological results was done.

\section{Statistical analysis}

Collected data was entered in MS excel and analysed using SPSS-19 version. Correlation of cytology and colposcopy with histopathology was done by applying the Chi Square test individually and $p$ value was calculated to study the statistical significance.

\section{RESULTS}

Total 80 women who were enrolled in the present study were categorized according to their demographic profile, high risk factors for carcinoma cervix (Table 1) clinical symptoms, clinical signs, cytology report, colposcopic findings, histopathology and finally the treatment they received. The study revealed that a large proportion of the women did not use any form of contraception (73.8\%). Among the contraceptive users, only $10 \%$ were using barrier form of contraception. $21.3 \%$ of cases had early age at first intercourse ( $<18$ years), $21.3 \%$ cases had poor personal hygiene, $6.3 \%$ cases had history of smoking, 72 cases $(90 \%)$ were multigravidas and 59 women $(73.8 \%)$ had unprotected sexual intercourse.

Table 1: The prevalence of high risk factors for carcinoma cervix.

\begin{tabular}{|lll|}
\hline High risk factors for CA cervix & $\mathbf{N}$ & $\%$ \\
\hline Early age at first intercourse (<18 years) & 17 & 21.3 \\
\hline Poor personal hygiene & 17 & 21.3 \\
\hline Smoking & 5 & 6.3 \\
\hline Multiple sexual partners & 0 & 0.0 \\
\hline Family history of CA cervix & 2 & 2.5 \\
\hline Multiparity & 72 & 90.0 \\
\hline Unprotected intercourse & 59 & 73.8 \\
\hline HPV DNA 16/18+ & 1 & 1.3 \\
\hline Comorbidities (DM and HTN) & 11 & 13.8 \\
\hline
\end{tabular}

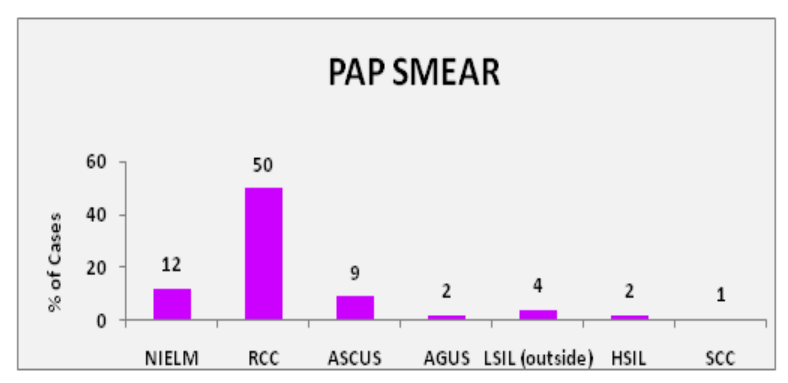

Figure 1: Distribution of women according to cytology report.

The most common clinical presentation was vaginal discharge seen in $68.8 \%$ women followed by menstrual disturbance seen in $26.3 \%$ women. The proportion of women with post coital bleed and intermenstrual bleeding was $12.5 \%$ each. On clinical examination, unhealthy vaginal discharge was present in $25 \%$ women while $23.8 \%$ women had a cervix which bled on touch. Erosion was uniformly present in all women as that was the inclusion criteria. On subjecting the women with cervical erosion to Pap smear, 12 of them $(15 \%)$ had a normal smear, $62.5 \%$ had inflammatory cells, $11.3 \%$ had ASCUS, 2.5\% had HSIL and AGUS, 5\% had LSIL and 
$1.3 \%$ had SCC. On subjecting the women to colposcopy, $12.5 \%$ had grade 2 aceto-white areas, $92.5 \%$ had negative iodine uptake and $26.2 \%$ had abnormal vasculature. 12 women had a modified RCI score of $\geq 6$. Out of these 3 had HSIL and 1 had SCC. Hence, modified RCI score of $\geq 6$ is highly suggestive of high-grade cervical lesions. Out of the 6 women with positive ridge sign, $2(33.3 \%)$ had CIN2/3 on histopathology.

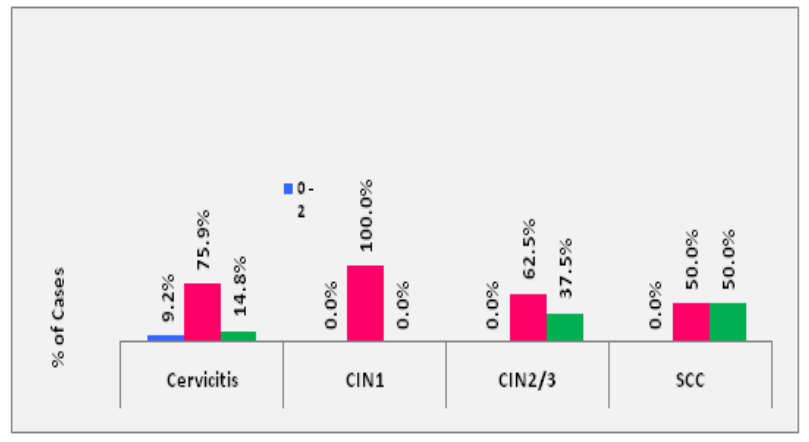

Figure 2: Correlation of colposcopic diagnosis and histopathology.

In the 4 women with positive rag sign, 1 (25\%) had CIN2/3 and $2(50 \%)$ had SCC. Among the 2 women with positive Inner border sign, $1(50 \%)$ had SCC on histopathology. Thus, presence of any of these colposcopic signs is highly indicative of high grade cervical lesions. Statistical analysis was carried out by calculating sensitivity, specificity, positive predictive value and negative predictive value for both cytology and colposcopy.

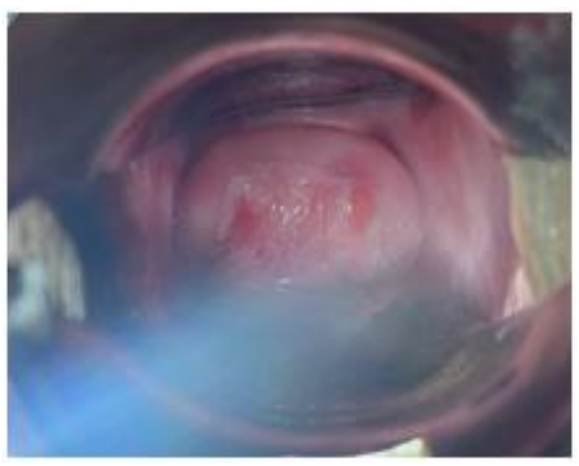

Figure 3: Acetowhitening.

It was concluded that sensitivity of colposcopy was significantly higher $(84.6 \%)$ as compared to cytology $46.1 \%$ implying that the patients testing positive for the disease truly had the disease. There were statistically insignificant number of patients who had cervical cancer and were missed on colposcopy. The specificity which denotes the truly negative cases was comparable in both cytology $(83.5 \%)$ and colposcopy but was higher in colposcopy $(86.5 \%)$. Also, the negative predictive value of colposcopy in this study was $96.6 \%$ signifying that patients having a negative screening by colposcopy were truly negative for the disease. There were $55 \%$ patients screened positive for cervical cancer on colposcopy who truly had the disease. However, the positive predictive value of cytology alone was significantly low (35.2\%) in this study. Chi square test was applied to the final results based on which the $\mathrm{p}$ value was calculated to be 0.000 making it statistically significant $(<5 \%)$. Hence, colposcopy and histopathology are dependent on each other implying that a colposcopic report suggestive of some degree of malignancy has a clinically significant probability of the histopathology report confirming it.

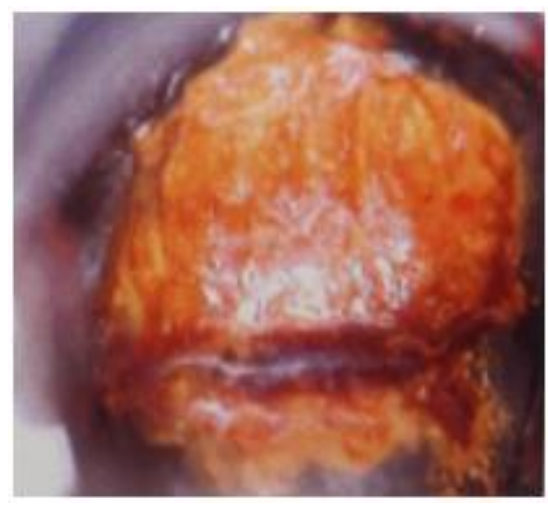

Figure 4: Negative iodine uptake.

\section{DISCUSSION}

The mean age in the present study was $36.18 \pm 8.2$ years. It was comparable to the study by Sharma et al and Pankaj et al in which the most common age group was 31-40 years. The mean parity in the present study was $2.78 \pm 1.39$ which was comparable to the studies by Sharma et al, Paswan et al and Bhalerao et al. ${ }^{4-7}$ The most common presenting complaint in this study was vaginal discharge as seen in $68.8 \%$ patients. This was comparable to studies by Goldacre et al, Paswan et al, Bhalerao et al and Garg et al where the most common presenting complaint was consistently vaginal discharge which may or may not be associated with lower backache..$^{6-9}$ In a study by Dasari et al unhealthy vaginal discharge was observed in $13.3 \%$ patients which was comparable to $25 \%$ patients presenting with unhealthy vaginal discharge in the present study. ${ }^{10}$ This increased incidence of unhealthy vaginal discharge may be attributed to the increased prevalence of lower socioeconomic strata and poor personal hygiene in our study group.

In the present study, the proportion of women having grade 2 acetowhite areas was $12.5 \%$ which was comparable to $12.3 \%$ in the study by Barut et al. ${ }^{11}$ The acetowhite lesions were found to be higher in the study by Garg et al and Joshi et al which may be explained as they took into account grade 1 flat acetowhite lesions. ${ }^{9,12}$ The proportion of women having MRCI > 6 in the present study was comparable with other studies by Sharma et al and Asmita et al. ${ }^{4,13}$ In the present study, the proportion of women having chronic cervicitis on histopathology was $67.5 \%$ which is comparable to the incidence in the 
studies by Garg et al (55\%), Barut et al (51.8\%) and Joshi

et al $(48 \%){ }^{9,12}$

Table 2: Diagnostic accuracy of cytology and colposcopy.

\begin{tabular}{|lllll|}
\hline Parameters & Sensitivity $(\%)$ & Specificity $(\%)$ & PPV $(\%)$ & NPV $(\%)$ \\
\hline Cytology & 46.1 & 83.5 & 35.2 & 88.8 \\
\hline Colposcopy & 84.6 & 86.5 & 55 & 96.6 \\
\hline
\end{tabular}

\section{Limitations}

A convenient sample size was chosen so that a thorough evaluation of patients could be carried out in a single visit and none are lost to follow up. However, larger samples and more studies are required to determine the optimal screening tool for cervical cancer. Moreover, colposcopy requires expensive equipment and training which is not available in limited resource settings and rural areas where there is prevalence of a large proportion of women exhibiting high risk factors for cervical cancer.

\section{CONCLUSION}

Amongst the 80 women who were studied, $10 \%$ of women harboured HSIL and $2.5 \%$ had SCC on histopathology. Hence, women with symptomatic cervical erosion should be thoroughly evaluated and cytology alone cannot rule out underlying high grade lesion. Colposcopic examination should ideally be carried out in symptomatic cervical erosion as it has higher sensitivity than cytology and also has a high negative predictive value. A colposcopic directed biopsy should be done to exclude malignancy in patients who have a high MRCI score on colposcopy or any of the colposcopic signs. Hence, we can conclude that a combination of cytology and colposcopy, whenever available, forms a more effective screening tool than cytology alone. Strengthening the screening process at the grass root level will ensure early detection and timely management of pre-malignant cervical lesions in the future.

\section{ACKNOWLEDGEMENTS}

Authors would like to thank all members of department of obstetrics and gynaecology in LHMC for their cooperation and support.

\section{Funding: No funding sources}

Conflict of interest: None declared

Ethical approval: The study was approved by the Institutional Ethics Committee

\section{REFERENCES}

1. Bruni L, Barrionuevo-Rosas L, Albero G, Aldea M, Serrano B, Valencia S, Brotons M, Mena M, Cosano $\mathrm{R}$, Muñoz J, Bosch FX. ICO information centre on HPV and cancer (HPV information centre). Human papillomavirus and related diseases in India. J Clin Epidemiol. 2015;72:12-23.

2. Burkett BJ, Peterson CM, Birch LM, Brennan C,
Nuckols ML, Ward BE, Crum CP. The relationship between contraceptives, sexual practices, and cervical human papillomavirus infection among a college population. J Clin Epidemiol. 1992;45(11):1295-302.

3. Srivastava AN, Misra JS, Srivastava S, Das BC, Gupta S. Cervical cancer screening in rural India: Status \& current concepts. Indian J Med Res. 2018; 148(6):687.

4. Sharma S, Saini M, Saini A, Bhalla AA. Colposcopic evaluation of patients with abnormal cervical cytology and its histopathological correlation: an original article. J Gynecol Obstet. 2014;18(1):1-8.

5. Pankaj S, Nazneen S, Kumari S, Kumari A, Kumari A, Kumari J, et al. Comparison of conventional Pap smear and liquid-based cytology: A study of cervical cancer screening at tertiary care center in Bihar. Indian J Cancer. 2018;55(1):80.

6. Paswan A. VIA (visual inspection with acetic acid) and VILI (visual inspection with lugol's iodine) as an initial approach with colposcopy as a next screening tool with its positive predictive value in low socioeconomic patients. Int $\mathrm{J}$ Reprod Contracept Obstet Gynecol. 2017;7(1):210-4.

7. Bhalerao A, Kulkarni S, Ghike S, Kawathalkar JS. Correlation of pap smear, colposcopy and histopathology in women with unhealthy cervix. J South Asian Feder Obst Gynaecol. 2012;4(2):97-8.

8. Goldacre MJ, Loudon N, Watt B, Grant G, Loudon JD, McPherson K, Vessey MP. Epidemiology and clinical significance of cervical erosion in women attending a family planning clinic. Br Med J. 1978; 1(6115):748-50.

9. Garg R, Desai R. Cytologic and colposcopic evaluation of all symptomatic women at tertiary care centre. Int J Adv Med. 2017;4(3):799.

10. Papa Dasari, S Rajathi, Surendra V Kumar, Colposcopic evaluation of cervix with persistent inflammatory Pap smear: A prospective analytical study, cytojournal. 2010;7:16.

11. Barut MU, Kale A, Kuyumcuoğlu U, Bozkurt M, Ağaçayak E, Özekinci S, Gul T. Analysis of sensitivity, specificity, and positive and negative predictive values of smear and colposcopy in diagnosis of premalignant and malignant cervical lesions. Medical science monitor. Int Med $\mathrm{J}$ Experiment Clin Res. 2015;21:3860.

12. Joshi C, Kujur P, Thakur N. Correlation of Pap smear and colposcopy in relation to histopathological findings in detection of premalignant lesions of cervix in a tertiary care centre. Int J Sci Stud. 2015;3(8):5560.

13. Ashmita D, Shakuntala PN, Rao SR. Comparison and correlation of PAP smear, colposcopy and 
histopathology in symptomatic women and suspicious looking cervix in a tertiary hospital care centre. Int $\mathbf{J}$ Health Sci Res. 2013;3(5):50-9.

Cite this article as: Goel S, Lal P. Concurrent cytologic and colposcopic evaluation of symptomatic cervical erosion. Int J Reprod Contracept Obstet Gynecol 2022;11:125-9. 